Research Paper

\title{
In Vivo Lymphatic Imaging of a Human Inflammatory Breast Cancer Model
}

\author{
Germaine D. Agollah ${ }^{1,2}$, Grace Wu${ }^{1}$, Eva M. Sevick-Muraca1 ${ }^{1}$, Sunkuk Kwon ${ }^{\circledR}$ \\ 1. Center for Molecular Imaging, The Brown Foundation Institute of Molecular Medicine, The University of Texas Health Science Center, \\ Houston, TX 77030; \\ 2. The University of Texas Graduate School of Biomedical Sciences at Houston. The University of Texas MD Anderson Cancer Center, \\ Houston, Texas 77030.
}

\begin{abstract}
$\square$ Corresponding author: Sunkuk Kwon, Ph.D., University of Texas Health Science Center at Houston, 1825 Pressler St, SRB 330F, Houston, TX 77030.

(C) Ivyspring International Publisher. This is an open-access article distributed under the terms of the Creative Commons License (http://creativecommons.org/ licenses/by-nc-nd/3.0/). Reproduction is permitted for personal, noncommercial use, provided that the article is in whole, unmodified, and properly cited.
\end{abstract}

Received: 2014.06.06; Accepted: 2014.09.25; Published: 20I4.10.23

\begin{abstract}
Background: Inflammatory breast cancer (IBC) remains the most aggressive type of breast cancer with the greatest potential for metastasis and as a result, the highest mortality rate. IBC cells invade and metastasize through dermal lymphatic vessels; however, it is unknown how lymphatic drainage patterns change during IBC growth and metastasis. Herein, we non-invasively and longitudinally imaged lymphatics in an animal model of IBC using near-infrared fluorescence (NIRF) imaging.

Materials and methods: Mice were imaged in vivo prior to, and up to II weeks after subcutaneous or orthotopic inoculation of human IBC SUMI 49 cells, which were stably transfected with infrared fluorescence protein (iRFP) gene reporter (SUMI49-iRFP), following intradermal (i.d.) injection of indocyanine green (ICG).

Results: Fluorescence images showed well-defined lymphatic vessels prior to SUMI49-iRFP inoculation. However, altered lymphatic drainage patterns including rerouting of lymphatic drainage were detected in mice with SUMI49-iRFP, due to lymphatic obstruction of normal lymphatic drainages caused by tumor growth. In addition, we observed tortuous lymphatic vessels and extravasation of ICG-laden lymph in mice with SUMI49-iRFP. We also observed increased and dilated fluorescent lymphatic vessels in the tumor periphery, which was confirmed by ex vivo immunohistochemical staining of lymphatic vessels.
\end{abstract}

Conclusions: Our pre-clinical studies demonstrate that non-invasive NIRF imaging can provide a method to assess changes in lymphatic drainage patterns during IBC growth and metastasis.

Key words: inflammatory breast cancer, near-infrared fluorescence imaging, lymphatic system, lymphangiogenesis.

\section{Introduction}

Inflammatory breast cancer (IBC) is the most aggressive, but poorly understood type of breast cancer. IBC constitutes up to $5 \%$ of all newly diagnosed breast cancers, yet responsible for $8-10 \%$ of breast cancer-related deaths [1]. IBC has a 15-year survival rate between $20-30 \%$ [2-4]. Approximately $40 \%$ of IBC patients have distant metastases to the brain, bones, and lymph nodes (LNs) at the time of diagnosis, as compared to $5 \%$ of patients with non-IBC [5]. Unfortunately, there are no definitive molecular or pathological diagnostic criteria for IBC [6]. IBC grows in nests or sheets, spreading outward into the skin and eventually distant sites through dermal lymphatic vessels. Therefore, one of the key 
pathological characteristics of IBC is dermal lymphatic invasion by tumor emboli, which can lead to obstruction of lymphatic drainage possibly causing the clinical inflammatory features of diffuse erythema, rapid breast enlargement and edema as indicated by peau d'orange (orange peel) appearance covering at least a third of the breast surface [7].

It has been demonstrated that dermal lymphatic involvement is associated with a high risk for local recurrence [8]. However, owing to the inability to directly image the lymphatics in vivo, the association between IBC metastasis and pre-existing lymphatic vasculature and/or tumor lymphangiogenesis has been assessed by indirect pathological evaluation of skin punch biopsies, which can be prone to tissue sampling error leading to missed diagnoses of dermal lymphatic involvement [6, 8]. Yet despite the important role of lymphatics as a critical pathway for cancer metastasis, relatively little is known about the role of lymphatic function in IBC growth and metastasis. Therefore, functional lymphatic imaging could shed light on the general mechanism of lymphovascular invasion exhibited by nearly all metastasizing cancers and ultimately result in novel therapeutics to arrest metastasis.

Our group has developed dynamic near-infrared fluorescence (NIRF) imaging techniques to image normal lymphatic function and drainage patterns in normal mice; functional and architectural lymphatic changes in mice with tumor metastasis, inflammation, or wound healing; and transgenic mice with a lymphatic phenotype following intradermal (i.d.) injection of a NIR fluorophore, indocyanine green (ICG) [9-14]. In addition, our group successfully imaged tumor growth and metastasis in an orthotopic prostate model in mice using far red fluorescence arising from a stably expressed gene reporter, infrared fluorescence protein (iRFP) $[15,16]$. The purpose of this study was to non-invasively and longitudinally image changes of lymphatic drainage patterns in mice bearing the triple-negative, human IBC SUM149 cells, which were stably transfected with iRFP gene reporter (SUM149-iRFP), in order to better monitor tumor growth and metastasis. Herein, we successfully imaged iRFP fluorescence during tumor growth and LN metastasis, and NIRF lymphatic imaging data showed a lymphatic phenotype in response to IBC growth. Thus, our findings suggest that longitudinal NIRF lymphatic imaging and far red fluorescence gene reporter can be used to assess lymphatic vessel network remodeling during tumor growth and metastasis in IBC.

\section{Materials and methods \\ Cells}

The human IBC cell line SUM149, which has been reported to have metastatic potential $[17,18]$ was obtained from Asterand (Detroit, MI). To generate a SUM149 cell line constitutively expressing the iRFP reporter gene for the mouse model, cells were cultured as monolayer in complete medium (Ham's-F12 containing $5 \%$ fetal bovine serum, 10 mM HEPES, 5 $\mathrm{ug} / \mathrm{ml}$ insulin, and $1 \mathrm{ug} / \mathrm{ml}$ hydrocortisone). A plasmid piRFP (Addgene, Cambridge, MA) carrying the iRFP reporter gene was transfected into the cells using Lipofectamine 2000 reagent (Invitrogen, Grand Island, NY ) according to manufacturer's directions. Transfected cells were carried under G418 selection in complete medium; survivors with top $5-10 \%$ iRFP intensity were sorted and used for subsequent experiments.

\section{Animal models of IBC}

Animals were maintained in a pathogen-free mouse facility accredited by the American Association for Laboratory Animal Care. All experiments were performed in accordance with guidelines of the Institutional Animal Care and Use Committee. Animal experiments were approved by University of Texas Health Science Center Institutional Animal Care and Usage Committee. Eight to ten weeks old female non-obese diabetic/severe combined immunodeficiency (NOD/SCID) mice (Charles River, Wilmington, MA) were housed and fed special alfalfa-free sterilized pelleted food (AIN-93G; OpenSource Diets, New Brunswick, NJ) in order to reduce diet-induced fluorescence in the abdomen [19] and sterilized water. Two weeks after feeding special diets, mice were injected with $5 \times 10^{6}$ SUM149-iRFP cells in $0.1 \mathrm{ml}$ PBS either subcutaneously in the left hindlimb or orthotopically in the left inguinal mammary fat pad (MFP).

\section{NIRF imaging and data analysis}

Animals were clipped and residual hair removed with depilatory cream (Nair, Church \& Dwight Co., Inc.) $24 \mathrm{hrs}$ before imaging. Mice were imaged for baseline information prior to inoculation of SUM149-iRFP cells. Anesthetized mice with isoflurane (2\% oxygen) were maintained on a warming pad at $37^{\circ} \mathrm{C}$. A volume of $10 \mu \mathrm{l}$ of $645 \mu \mathrm{M}$ of ICG (Akorn, Inc.) dissolved in mixture of distilled water and $0.9 \%$ sodium chloride in a volume ratio of 1:9 was injected intradermally to the base of the tail using with 31 gauge needles (BD). Fluorescence images were acquired immediately after and for up to $20 \mathrm{~min}$ after i.d. injection using our custom-built far-red and NIRF imaging systems as described previously [9-15]. To 
achieve a greater magnification, a macrolens (Infinity K2/SC video lens, Edmund Optics Inc.) was used.

Matlab (The MathWorks, Inc., Natick, MA) and ImageJ (National Institutes of Health, Washington, DC) were used to analyze the fluorescence imaging data in the following manner. To reveal lymphatic contractility, fixed regions of interest (ROIs) in fluorescent lymph channels were defined on fluorescence images [14]. The mean of the fluorescence intensity within each ROI in each fluorescence image was then calculated and plotted as a function of imaging time.

\section{Immunohistochemical (IHC) staining}

Skin samples were fixed in $10 \%$ formalin overnight before transfer into $70 \%$ ethanol and embedding in paraffin. $4 \mu \mathrm{m}$ sections were used in all staining procedures which included hematoxylin-eosin (H\&E). For LYVE-1 detection, following paraffin removal and antigen retrieval using citrate buffer $\mathrm{pH}$ 6, tissues were blocked with $5 \%$ bovine serum albumin (BSA) and stained with rabbit anti-mouse LYVE-1 antibody (AngioBio) and biotin-anti rabbit secondary antibody (Vector Labs). VectaStain Elite ABC peroxidase detection system and $\mathrm{DAB}$ chromagen were used before tissues were counter-stained with hematoxylin (Vector Labs). LYVE-1 expression was examined at $x 40$ magnification (Leica Microsystems Inc.). Tissues were also stained with Cytokeratin 8 antibody (Abcam) and
Alexa Fluor $594 \mathrm{~nm}$ secondary antibody (Life Technologies) and counterstained with DRAQ5 (BioStatus). ImageJ software (NIH) was used to analyze the number of lymphatic vessels and percentage of positively stained lymphatic vessels per area was determined.

\section{Statistical analysis}

Data were presented as average values \pm standard error. Statistical analysis was performed using SigmaPlot 11.0 (Systat Software, Inc). The two-sample $t$ test was used and the differences were considered significant at $\mathrm{p}<0.05$.

\section{Results}

SUM149 cells were transfected with piRFP plasmid to obtain fluorescent cells. After transfection, cells were selected with medium that contained 1200 $\mu \mathrm{g} / \mathrm{mL}$ gentamicin (G418). Subclones with high iRFP-expression were isolated in 96-well plates by limiting dilution. iRFP expression was confirmed by immunofluorescence (Figure 1A) and FACs analysis (Figure 1B). There was no difference in the cell proliferation rates of parental cells and selected RFP transfectants, as determined by comparing their growth rate in monolayer culture (data not shown).

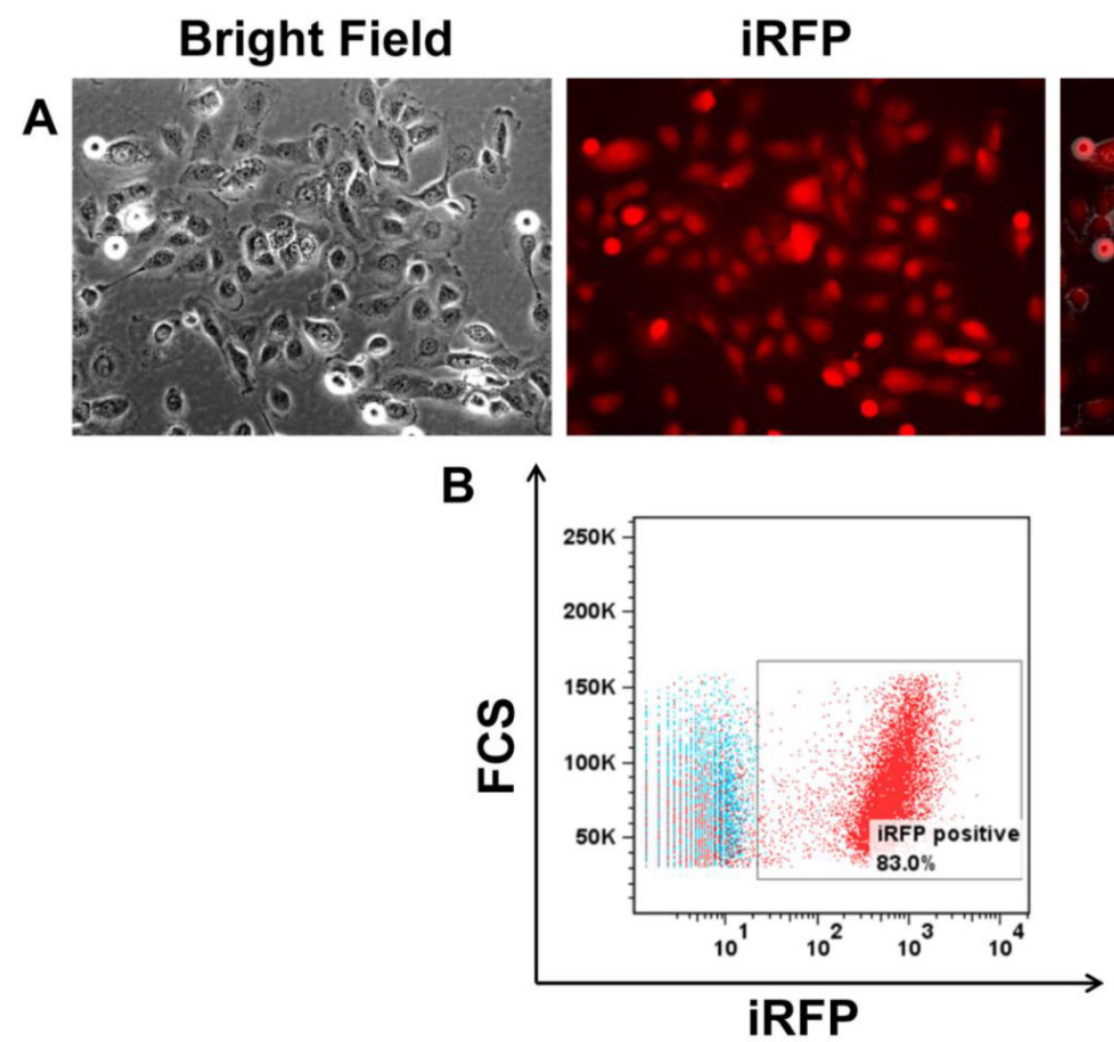

Figure I. (A) Bright field, iRFP fluorescence, and an overlay of SUMI49 transfected with iRFP showing red cytoplasmic fluorescent signal. (B) FACS analysis showing $>80 \%$ iRFP expression in SUMI 49. Scale, $200 \mu \mathrm{m}$. 
SUM149-iRFP cells were orthotopically inoculated in the MFP of mice and visualized longitudinally over nine weeks following implantation (Figure 2). In conjunction with tumor growth assessment, we also performed lymphatic imaging to investigate how lymphatic drainage patterns change during disease progression. As reported previously [9, 10], ICG-laden lymph drained from the base of the tail, where ICG was injected, to the ILN and to the ALN. This drainage pathway was detected in nearly all mice imaged in this study, with the exception of one mouse (See Figure 3). Normal lymphatic drainage pathways to the ALN were observed up to 3 weeks post inoculation (p.i.), even as ICG accumulated proximally to the tumor. At 5 weeks p.i., we observed strong ICG fluo- rescence in the proximity of the tumor. Magnified fluorescent images acquired using a macrolens showed lymphatic capillary network (open arrow in Figure 2). Dynamic NIRF imaging (Additional file 1: video 1) demonstrated ICG dye accumulated anteriorly to the tumor, which exhibited both lateral and retrograde flow due to obstruction of normal lymphatic drainage towards the ILN by the tumor. At 7 weeks p.i, these anterior vessels were no longer observed; rather, new drainage pathways to the ALN had formed which bypassed both the tumor and tumor draining ILN (TdILN). By 9 weeks p.i., we observed rerouting of ICG-laden lymph to the ALN and the more tortuous and leaky lymphatic vessels (Additional file 2: video 2) as tumor burden increased.

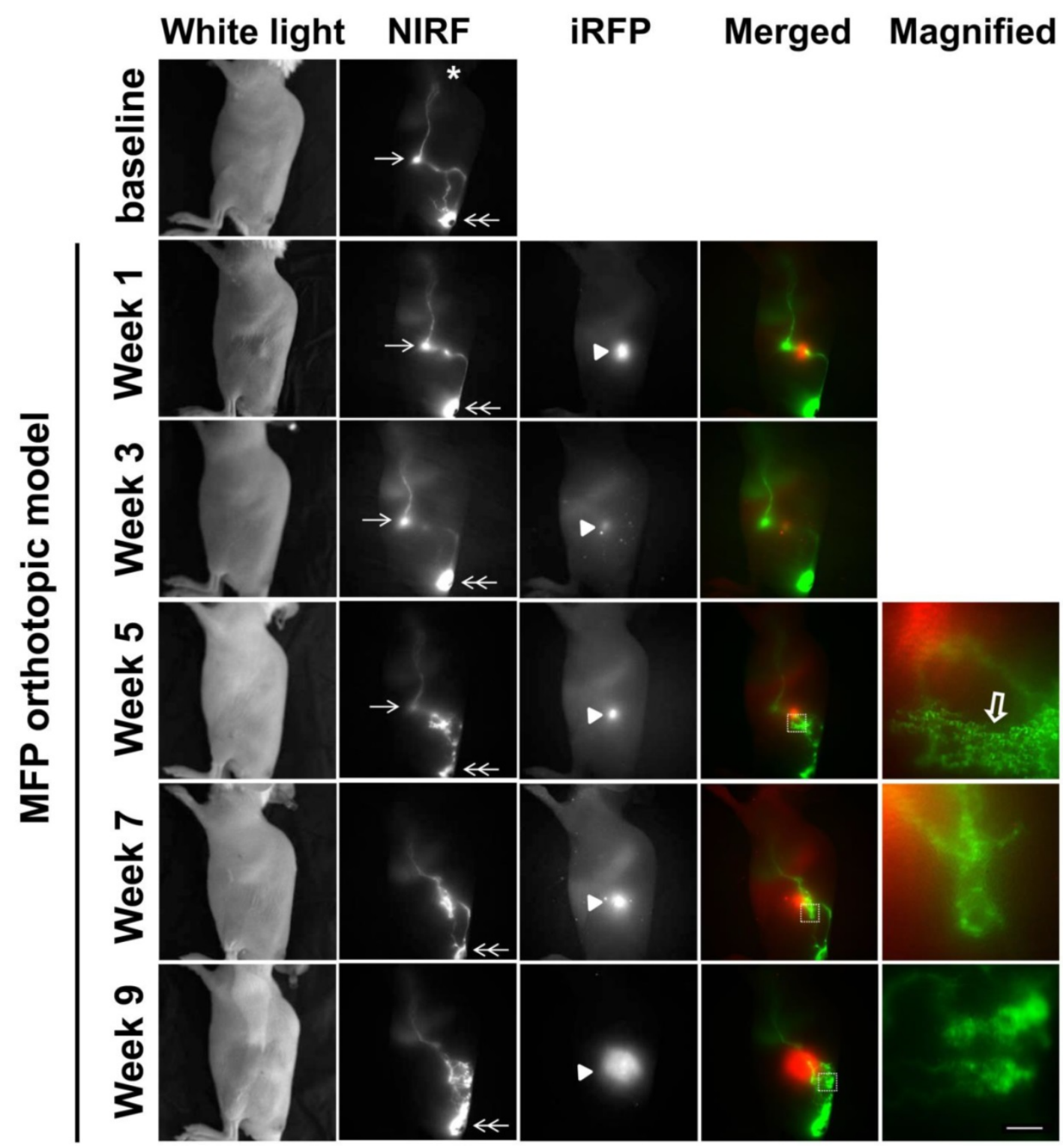

Figure 2. White light, NIR, and iRFP fluorescent images prior to and for up to 9 weeks after orthotopic inoculation of SUMI49-iRFP cells to the inguinal MFP. Images were acquired 10 mins after i.d. injection of ICG. NIR fluorescent images (green) of the lymphatics were merged with iRFP fluorescent images (red) of the gene reporters in the tumor. Magnified fluorescent images of the white rectangles were also acquired. Arrow, ILN. Double arrow, injection site. Arrow head, SUMI49-iRFP tumor. Open arrow, lymphatic capillaries due to dermal backflow. Asterisk, ALN. Scale, I mm. 


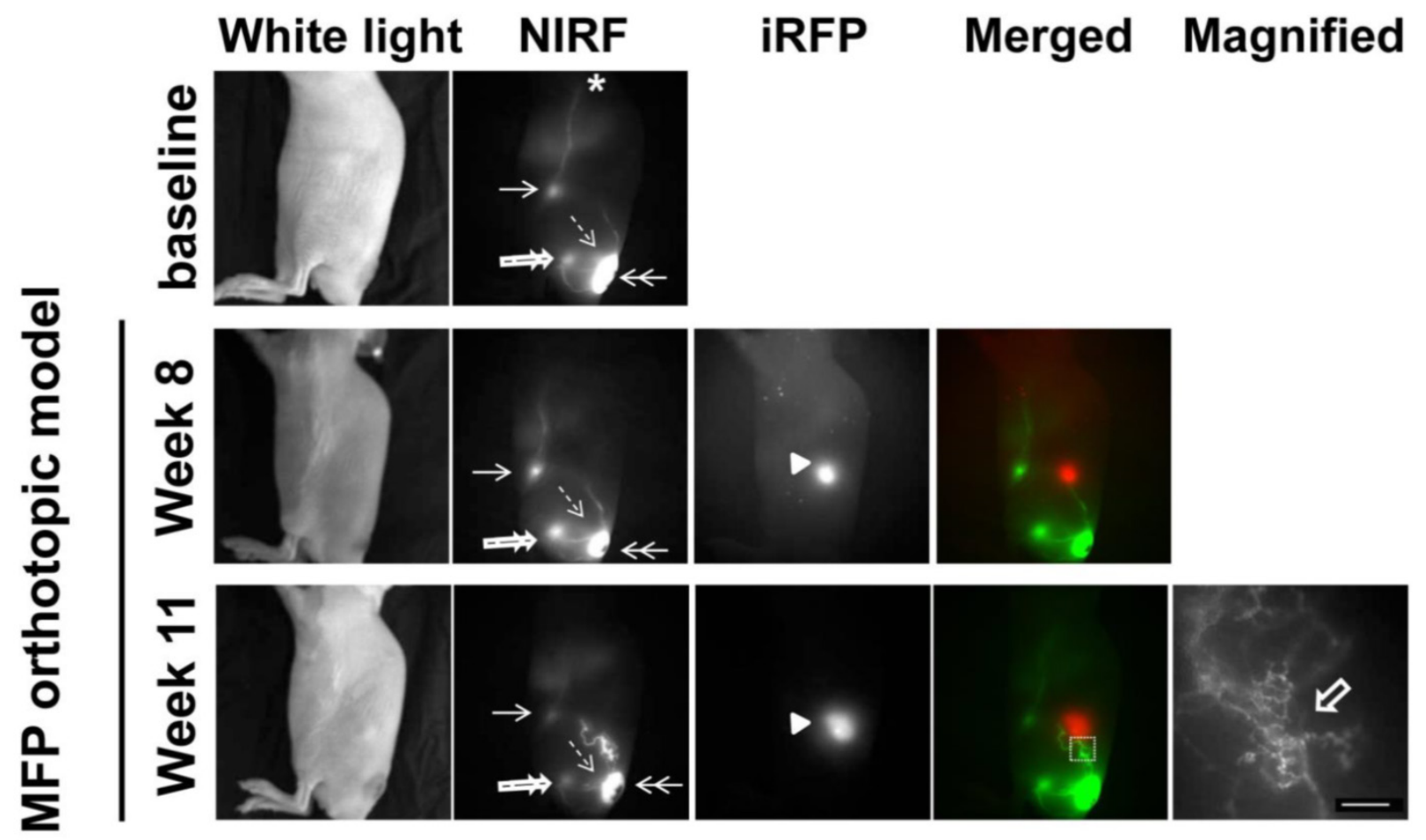

Figure 3. White light, NIR, and iRFP fluorescent images prior to, 8 weeks, and I I weeks after orthotopic inoculation of SUMI49-iRFP cells to the inguinal MFP. NIR fluorescent images (green) of the lymphatics were merged with iRFP fluorescent images (red) of the gene reporter in the tumor. A magnified fluorescent image of the white rectangles was also acquired. Arrow, ILN. Double arrow, ICG injection site. Double open arrow, PLN. Broken arrow, IsLN. Arrow head, SUMI49-iRFP tumor. Open arrow, lymphatic capillaries due to dermal backflow. Scale, I mm.

As previously mentioned, one mouse showed altered lymphatic drainage prior to orthotopic inoculation of tumor cells (Figure 3). The baseline images from this mouse showed lymphatic drainage from the injection site (double arrow) to the popliteal LN (PLN) (double open arrow) and then to the ischial LN (IsLN) (broken arrow) and to the ILN (arrow) and then to the ALN (asterisk, Additional file 3: video 3). At 11 weeks p.i., we detected rerouting around the orthotopic tumor in comparison to 8 weeks p.i., due to tumor obstruction of normal lymphatic drainage towards the ILN. We also observed dermal backflow into the lymphatic capillary network shown in the magnified image. However, there were no alterations in the drainage patterns from ICG injection site to the PLN and IsLN and from ILN to the ALN.

To assess how a different implantation model would affect lymphatic drainage, we also performed subcutaneous (s.c) tumor implantation and longitudinally monitored tumor growth and changes in lymphatic drainage (Figure 4). NIRF imaging showed ICG-laden lymph draining along the lymphatic vessels in the skin above the tumor at 1 week p.i., with increased fluorescent intensity and fluorescent spots. At 3 weeks after inoculation, we observed ICG accu- mulation in the tumor as evidenced by strong fluorescence likely due to leaky lymphatic vessels (Additional file 4: video 4). However, ICG intratumoral accumulation was not detected at 4 weeks p.i.; instead, we observed dermal backflow proximal to the tumor. At 5 weeks p.i. alternate lymphatic routes were observed around the tumor which were dilated by 7 weeks p.i; and ICG accumulated anteriorly around the tumor margin at week 9 . Furthermore, our in vivo imaging data also showed iRFP fluorescence in the TdILN in this mouse, indicative of LN metastasis (Figure 5A) and was confirmed by ex vivo intravital iRFP-fluorescence imaging of the TdILN. SUM149 overexpresses luminal cytokeratin 8 [20]; therefore, we further confirmed local LN metastasis by IHC staining and detected cytokeratin-positive cells in the TdILN compared to the contralateral ILN (Figure 5B).

Since we observed an increased number of dilated fluorescent lymphatic vessels in the periphery tumors in vivo (Figures 2 and 4), we also performed IHC staining of lymphatic vessels using LYVE-1 antibody. As shown in Figure 6, there was a significantly increased number of peritumoral lymphatic vessels which occupied a greater area, and were thus more dilated, as compared to control skin. 


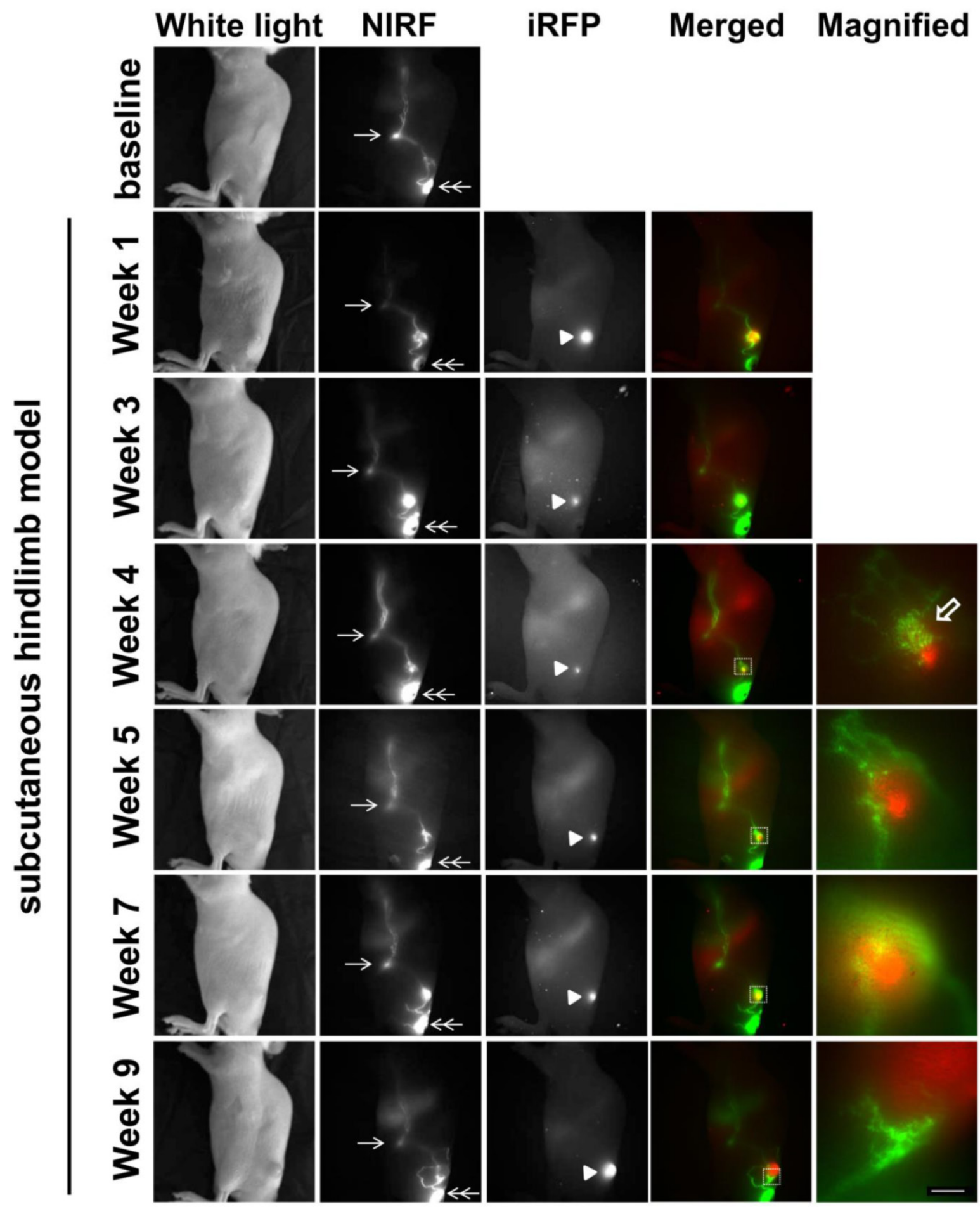

Figure 4. White light, NIR, and iRFP fluorescent images prior to and for up to 9 weeks after s.c. inoculation of SUMI49-iRFP cells. Images were acquired 10 mins after i.d. injection of ICG. NIR fluorescent images (green) of the lymphatics were fused with iRFP fluorescent images (red) of the gene reporters in the tumor. Magnified fluorescent images of the white rectangles were also acquired. Arrow, ILN. Double arrow, injection site. Arrow head, SUMI49-iRFP tumor. Open arrow, lymphatic capillaries due to dermal backflow. Scale, I mm. 


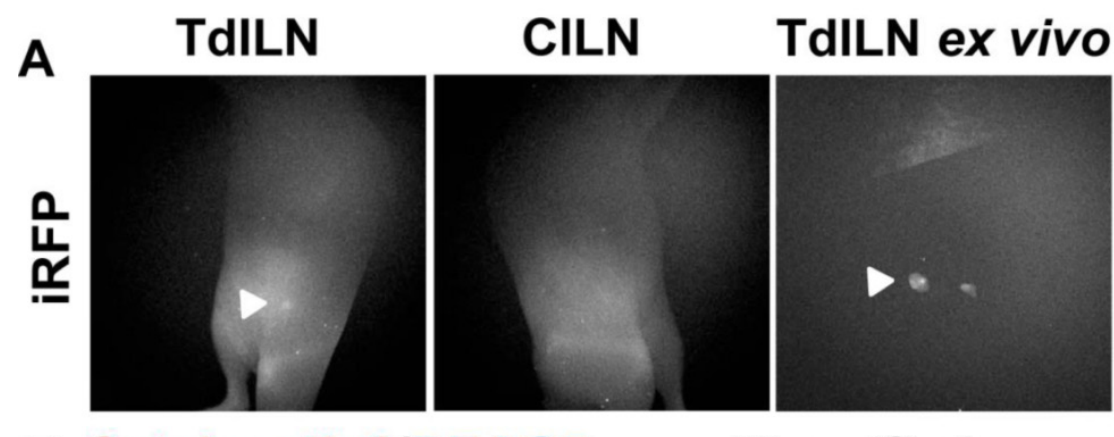

B Cytokeratin8/DRAQ5

Magnified
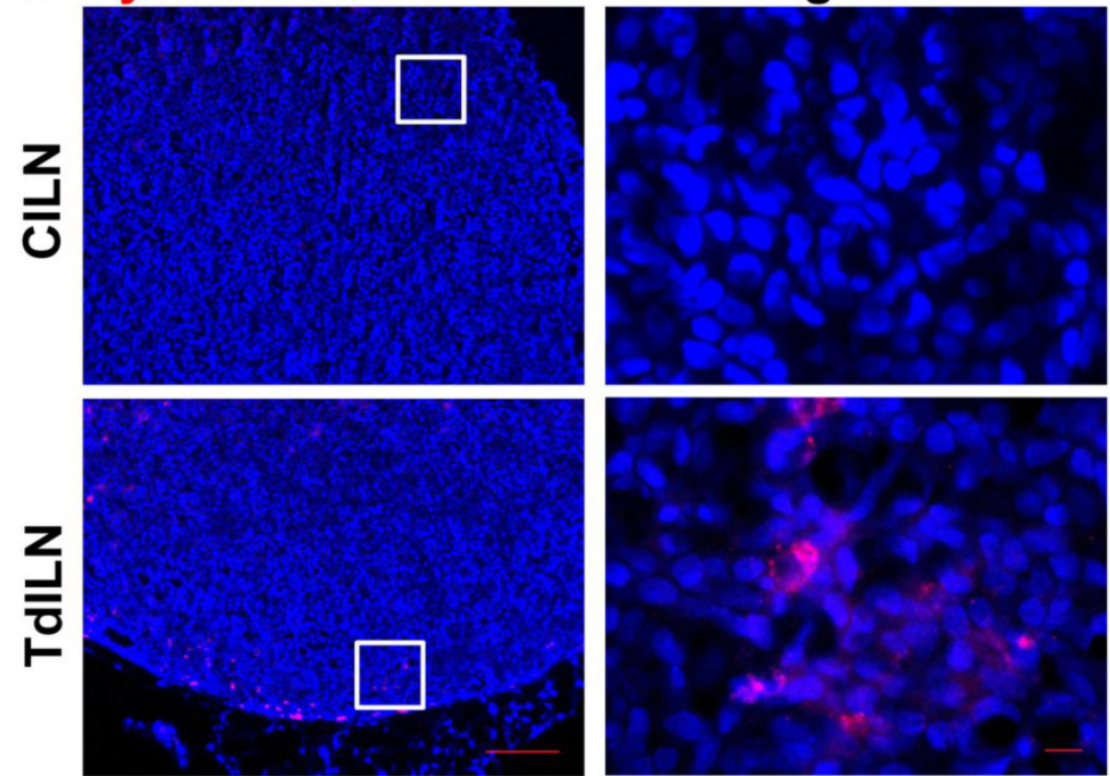

Figure 5. In vivo and ex vivo fluorescent images showing iRFP signal in the TdILN as compared to CILN, indicative of LN metastasis (A). IHC staining of cytokeratin 8 (red) also confirmed LN metastasis of SUMI49-iRFP (B). Blue, DRAQ5 nuclear stain. Arrow, TdILN. Scale, $100 \mu \mathrm{m}$ and I0 $\mu \mathrm{m}$ (magnified).

A Control

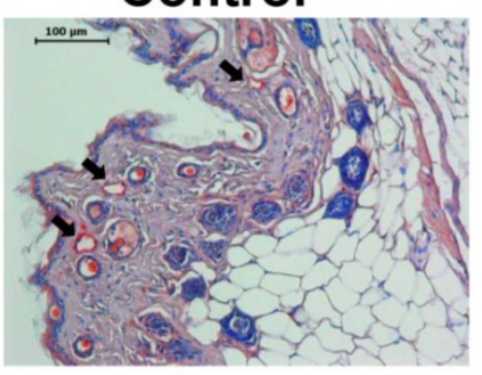

C

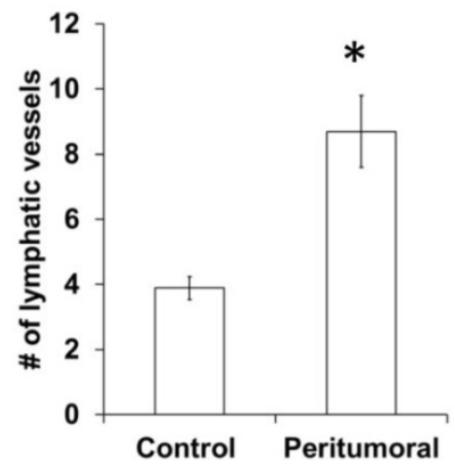

B

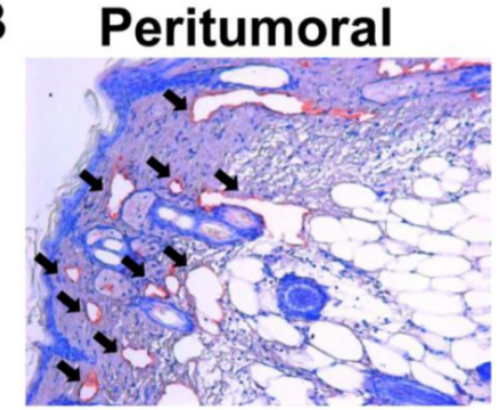

D

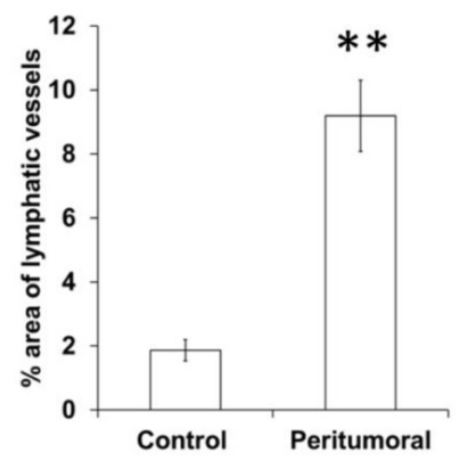

Figure 6. Immunohistochemical stain for LYVE-I (red staining; black arrows) showing more and dilated lymphatic vessels in the tumor periphery (B) as compared to the contralateral (control) region $(A)$. The number $(C)$ and relative area $(D)$ of peritumoral lymphatic vessels was significantly higher than contralateral skin tissues. $n=7$ mice. Scale, $100 \mu \mathrm{m} . * \mathrm{p}<0.05, * * \mathrm{p}<0.01$ vs. control. 


\section{Discussion}

Currently, MARY-X is the only pre-clinical model that recapitulates the lymphatic intravasation phenotype of human IBC $[6,21]$. MARY-X induces erythema in the skin over the tumor, mimicking the clinical presentation of IBC, and grows exclusively within murine lymphatic and blood vessels [21]. Another commonly used pre-clinical IBC model is SUM149, initially derived from a primary tumor of a patient with invasive ductal carcinoma [22]. SUM149 grows as multiple nodes/clusters and produces spontaneous local skin and/or distant metastasis to the leg after the axillary MFP injection [23]. In this study, we stably transfected iRFP gene reporter into SUM149 cells to enable longitudinal assessment of tumor growth and metastatic progression to LNs. Unlike other gene reporters such as GFP and DsRed, iRFP emits in the far-red region and is thus more suitable for in vivo imaging due to relatively low tissue absorption, scattering, and autofluorescence, resulting in increased light penetration [24]. However, in vivo iRFP fluorescence imaging requires animals be fed with special alfalfa-free diets to reduce fluorescence induced by standard murine diets that contain chlorophyll [15, 19]. Previously, similar to iRFP, IFP1.4 was also developed as another far-red fluorescent protein [25]. However, IFP1.4 requires exogenous administration of chromophore biliverdin to further emit fluorescence, whereas iRFP can use physiological levels of endogenous biliverdin to be visualized in tissues $[24,25]$.

Herein, we show that when iRFP fluorescence imaging is combined with functional ICG-based NIRF imaging, it provides important information about how the lymphatics change during tumor growth. Our NIRF imaging data showed gradual changes in lymphatic drainage patterns around the tumor. In mice with an orthotopic tumor (Figure 2), ICG accumulation was observed in the peritumoral region at 5 weeks p.i., indicative of obstruction of normal lymphatic drainage by the tumor. As shown in Additional file 1: Video 1, increased tumor burden resulted in obstruction of normal lymph flow, and eventually in dermal backflow anterior to the tumor. At week 7, rerouting of lymphatic drainage was underway to bypass both the tumor and TdILN, which was more evident by week 9 (Additional file 2: Video 2). Similarly, mice with subcutaneous hindlimb implantation also exhibited altered lymphatic drainage patterns during tumor progression (Figure 4). We observed that 6 out of 7 mice showed dermal lymphatic backflow and 5 of 7 mice collateral lymphatic vessels draining to the ILN by 9 weeks p.i.. Interestingly, as shown in Additional file 4: Video 4, extravasation of
ICG into SUM149-iRFP tumor was detected at 3 weeks p.i., due to leaky lymphatic vessels. Although lymphatic vessel function in the afferent inguinal lymphatic vessels was not quantified due to changes in lymphatic drainage pathways during tumor progression, we measured contraction frequency in the efferent inguinal collecting lymphatic vessels, which was not statistically different between baseline prior to tumor cell inoculation and 9 weeks p.i. $(n=6 ; 8.8 \pm$ 1.4 vs $7.8 \pm 0.8$ ).

Rerouting of tumor drainage during tumor growth and LN metastasis has been observed in clinical and preclinical studies [10, 14, 26-30]. These altered drainage patterns are due mainly to blockage of normal lymphatic drainage by the tumor, thus triggering alternate, collateral pre-existing lymphatic vessels to drain stagnant lymph to the non-sentinel LN. It has been reported that lymphatic drainage patterns in the skin are highly variable among patients, even with identical lesion sites, and therefore cannot be predicted [31]. A previous study demonstrated a significantly higher mRNA expression of lymphangiogenesis-related factors, such as vascular endothelial growth factor (VEGF)-C, in IBC than in non-IBC [32]. VEGF-C is a key lymphangiogenic factor known to induce tumor lymphangiogenesis and enhance tumor spread to the regional LNs and distant sites in mouse models of breast cancer [33-35]. Van der Auwera et al. demonstrated that intratumoral lymphatic vessels in IBC were larger and more numerous than those in non-IBC [36], which were often found to collapse due to growing tumor cells [37]. In addition, Van der Auwera et al. found that peritumoral lymphatic vessels in IBC had significantly higher lymphatic endothelial cell (LEC) proliferation than those in non-IBC, although there is no difference in LEC proliferation in intratumoral areas between IBC and non-IBC [36]. Taken together, remodeling of lymphatic vessels by active tumor lymphangiogenesis may also lead to changes in lymphatic drainage patterns and the propensity of IBC to metastasize via the lymphatic vasculature.

In conclusion, using NIRF lymphatic imaging with sufficient spatial (4.49 and 36 Line Pairs/mm for whole body and magnified imaging, respectively) and temporal (200 ms exposure time) resolution and sensitivity, we showed changes of lymphatic drainage patterns in mice subcutaneously or orthotopically inoculated with SUM149 human IBC cells that stably express a far-red excited gene reporter; however, additional work is required to determine whether a lymphatic phenotype we observed in mice bearing SUM149-iRFP is specific to IBC as compared to non-IBC. Nonetheless, dual-wavelength imaging of a combination of lymphatics (ICG excitation, $785 \mathrm{~nm}$; 
emission, $810 \mathrm{~nm}$ ) and tumor progression and metastasis (iRFP excitation, $690 \mathrm{~nm}$; emission, $710 \mathrm{~nm}$ ) used in this study enables a new method to non-invasively monitor lymphatic response to IBC growth and metastasis and assess longitudinal changes in response to new therapeutic strategies. Previously, we imaged atypical tortuous lymphatics and altered lymphatic drainage in the arm and affected breast of a patient with IBC using clinical NIRF technology to image as deep as 3-5 $\mathrm{cm}$ with a microdose of ICG [38-40]. Therefore, NIRF lymphatic imaging can be used for non-invasive assessment of lymphatic involvement of metastatic human IBC.

\section{Supplementary Material}

Additional File 1:

Video 1. ICG flow in a mouse 5 weeks after orthotopic inoculation of SUM149-iRFP cells to the inguinal MFP from Figure 2.

http://www.jcancer.org/v05p0774s1.avi

Additional File 2:

Video 2. ICG flow in a mouse 9 weeks after orthotopic inoculation of SUM149-iRFP cells to the inguinal MFP from Figure 2.

http://www.jcancer.org/v05p0774s2.avi

Additional File 3:

Video 3. ICG flow in a mouse prior to orthotopic inoculation of SUM149-iRFP cells from Figure 3.

http://www.jcancer.org/v05p0774s3.avi

Additional File 4:

Video 4. ICG flow in a mouse 3 weeks after s.c. inoculation of SUM149-iRFP cells from Figure 4.

http://www.jcancer.org/v05p0774s4.avi

\section{Acknowledgments}

The authors would like to thank Holly Robinson and Karen Gore for technical assistance with the mice used in these experiments and Dr. Melissa B. Aldrich for scientific review of manuscript. This work was supported in parts by National Institutes of Health R21 CA159293 (S.K), R01 CA128919 (E.M.S-M) and U54 CA136404 and (E.M.S-M). G.D.A acknowledges support from The Schissler Foundation Fellowship for Translational Studies of Common Human Diseases.

\section{Conflicts of Interest}

Dr. Sevick-Muraca declares financial interest in the NIRF Imaging, Inc., a company seeking to commercialize NIRF lymphatic imaging. No other conflicts of interest are declared for the remaining authors.

\section{References}

1. Hance KW, Anderson WF, Devesa SS. et al. Trends in inflammatory breast carcinoma incidence and survival: the surveillance, epidemiology, and end results program at the National Cancer Institute. J Natl Cancer Inst. 2005; 97: 966-75.

2. Levine PH, Steinhorn SC, Ries LG. et al. Inflammatory breast cancer: the experience of the surveillance, epidemiology, and end results (SEER) program. J Natl Cancer Inst. 1985; 74: 291-7.

3. Low JA, Berman AW, Steinberg SM. et al. Long-term follow-up for locally advanced and inflammatory breast cancer patients treated with multimodality therapy. J Clin Oncol. 2004; 22: 4067-74.

4. Ueno NT, Buzdar AU, Singletary SE. et al. Combined-modality treatment of inflammatory breast carcinoma: twenty years of experience at M. D. Anderson Cancer Center. Cancer Chemother Pharmacol. 1997; 40: 321-9.

5. Jaiyesimi IA, Buzdar AU, and Hortobagyi G. Inflammatory breast cancer: a review. J Clin Oncol. 1992; 10: 1014-24.

6. Robertson FM, Bondy M, Yang W. et al. Inflammatory breast cancer: the disease, the biology, the treatment. CA Cancer J Clin. 2010; 60: 351-75.

7. Greene FL, Page DL, Fleming ID. et al. AJCC Cancer Staging Manual. 6th ed. New York, NY: Springer-Verlag. 2003: 255-281.

8. Yamauchi $\mathrm{H}$, Woodward WA, Valero V. et al. Inflammatory breast cancer: what we know and what we need to learn. Oncologist. 2012; 17: 891-9.

9. Kwon S and Sevick-Muraca EM. Mouse phenotyping with near-infrared fluorescence lymphatic imaging. Biomed Opt Express. 2011; 2: 1403-1411.

10. Kwon $\mathrm{S}$ and Sevick-Muraca EM. Functional lymphatic imaging in tumor-bearing mice. J Immunol Methods. 2010; 360: 167-72.

11. Kwon $S$ and Sevick-Muraca EM. Noninvasive quantitative imaging of lymph function in mice. Lymphat Res Biol. 2007; 5: 219-31.

12. Lapinski PE, Kwon S, Lubeck BA. et al. RASA1 maintains the lymphatic vasculature in a quiescent functional state in mice. I Clin Invest. 2012; 122: 733-47.

13. Burrows PE, Gonzalez-Garay ML, Rasmussen JC. et al. Lymphatic abnormalities are associated with RASA1 gene mutations in mouse and man. Proc Natl Acad Sci U S A. 2013; 110: 8621-6.

14. Kwon S, Agollah GD, Wu G. et al. Direct visualization of changes of lymphatic function and drainage pathways in lymph node metastasis of B16F10 melanoma using near-infrared fluorescence imaging. Biomed Opt Express. 2013; 4: 967-77.

15. Zhu B, Wu G, Robinson H. et al. Tumor margin detection using quantitative NIRF molecular imaging targeting EpCAM validated by far red gene reporter iRFP. Mol Imaging Biol. 2013; 15: 560-8.

16. Lu Y, Darne CD, Tan IC. et al. In vivo imaging of orthotopic prostate cancer with far-red gene reporter fluorescence tomography and in vivo and ex vivo validation. J Biomed Opt. 2013; 18: 101305.

17. Kuperwasser C, Dessain S, Bierbaum BE. et al. A mouse model of human breast cancer metastasis to human bone. Cancer Res. 2005; 65: 6130-8.

18. Zbytek B, Carlson JA, Granese J. et al. Current concepts of metastasis in melanoma. Expert Rev Dermatol. 2008; 3: 569-585.

19. Kwon S, Davies-Venn C, and Sevick-Muraca EM. In vivo dynamic imaging of intestinal motions using diet-related autofluorescence. Neurogastroenterol Motil. 2012; 24: 494-7.

20. Wong SL, Balch CM, Hurley P. et al. Sentinel lymph node biopsy for melanoma: American Society of Clinical Oncology and Society of Surgical Oncology joint clinical practice guideline. J Clin Oncol. 2012; 30: 2912-8.

21. Alpaugh ML, Tomlinson JS, Shao ZM. et al. A novel human xenograft model of inflammatory breast cancer. Cancer Res. 1999; 59: 5079-84.

22. Forozan F, Veldman R, Ammerman CA. et al. Molecular cytogenetic analysis of 11 new breast cancer cell lines. Br J Cancer. 1999; 81: 1328-34.

23. Singh B, Cook KR, Martin C. et al. Evaluation of a CXCR4 antagonist in a xenograft mouse model of inflammatory breast cancer. Clin Exp Metastasis. 2010; 27: 233-40.

24. Filonov GS, Piatkevich KD, Ting LM. et al. Bright and stable near-infrared fluorescent protein for in vivo imaging. Nat Biotechnol. 2011; 29: 757-61.

25. Shu X, Royant A, Lin MZ. et al. Mammalian expression of infrared fluorescent proteins engineered from a bacterial phytochrome. Science. 2009; 324: 804-7.

26. Proulx ST, Luciani P, Christiansen A. et al. Use of a PEG-conjugated bright near-infrared dye for functional imaging of rerouting of tumor lymphatic drainage after sentinel lymph node metastasis. Biomaterials. 2013; 34: 5128-37.

27. Leijte JA, van der Ploeg IM, Valdes Olmos RA. et al. Visualization of tumor blockage and rerouting of lymphatic drainage in penile cancer patients by use of SPECT/CT. J Nucl Med. 2009; 50: 364-7.

28. Uren RF, Howman-Giles R, Chung DK. et al. Metastatic occlusion of a lymphatic collecting vessel in a patient with cutaneous melanoma and clinically normal lymph nodes. Clin Nucl Med. 2007; 32: 312-3.

29. Lam TK, Uren RF, Scolyer RA. et al. False-negative sentinel node biopsy because of obstruction of lymphatics by metastatic melanoma: the value of ultrasound in conjunction with preoperative lymphoscintigraphy. Melanoma Res. 2009; 19: 94-9.

30. Norman J, Cruse CW, Espinosa C. et al. Redefinition of cutaneous lymphatic drainage with the use of lymphoscintigraphy for malignant melanoma. Am J Surg. 1991; 162: 432-7.

31. Uren RF, Howman-Giles R, and Thompson JF. Patterns of lymphatic drainage from the skin in patients with melanoma. J Nucl Med. 2003; 44: 570-82.

32. Van der Auwera I, Van Laere SJ, Van den Eynden GG. et al. Increased angiogenesis and lymphangiogenesis in inflammatory versus noninflammatory breast cancer by real-time reverse transcriptase-PCR gene expression quantification. Clin Cancer Res. 2004; 10: 7965-71. 
33. Skobe $\mathrm{M}$, Hawighorst $\mathrm{T}$, Jackson DG. et al. Induction of tumor lymphangiogenesis by VEGF-C promotes breast cancer metastasis. Nat Med. 2001; $7:$ : 192-8

34. Mattila MM, Ruohola JK, Karpanen T. et al. VEGF-C induced lymphangiogenesis is associated with lymph node metastasis in orthotopic MCF-7 tumors. Int J Cancer. 2002; 98: 946-51.

35. Karpanen T, Egeblad M, Karkkainen MJ. et al. Vascular endothelial growth factor C promotes tumor lymphangiogenesis and intralymphatic tumor growth. Cancer Res. 2001; 61: 1786-90.

36. Van der Auwera I, Van den Eynden GG, Colpaert CG. et al. Tumor lymphangiogenesis in inflammatory breast carcinoma: a histomorphometric study. Clin Cancer Res. 2005; 11: 7637-42

37. Padera TP, Kadambi A, di Tomaso E. et al. Lymphatic metastasis in the absence of functional intratumor lymphatics. Science. 2002; 296: 1883-6.

38. Sevick-Muraca EM. Translation of near-infrared fluorescence imaging technologies: emerging clinical applications. Annu Rev Med. 2012; 63: 217-31.

39. Sevick-Muraca EM, Kwon S, and Rasmussen JC. Emerging lymphatic imaging technologies for mouse and man. J Clin Invest. 2014; 124: 905-14.

40. Meric-Bernstam F, Rasmussen JC, Krishnamurthy S. et al. Toward nodal staging of axillary lymph node basins through intradermal administration of fluorescent imaging agents. Biomed Opt Express. 2014; 5: 183-96. 